\title{
Über die Darstellung von amorphem Silicium, Siliciumsulfid, Siliciumchlorid und von Sulfosilikaten.
}

\author{
Von \\ WaLTher HeMpel und von HAast. ${ }^{1}$
}

Mit 1 Figur im Text.

Sehr viele Schlacken, die bei hüttenmännischen Prozessen auftreten, haben die Eigentümlichkeit, beim Zusammenkommen mit Hüssigem oder dampfförmigem Wasser Schwefelwasserstoff zu entwickeln. Diese Eigenschaft ist durch die gewöhnliche Annahme, dals diese Produkte Schwefelcalcium enthalten, nicht zu erklären, da dieses ein in Wasser unlöslicher und durch Wasser nicht zerlegbarer Körper ist, wie die Untersuchungen über das Verhalten der Sodarückstände des LE-BLANC-Verfahrens gelebrt haben.

Von dem Gedanken ausgehend, dafs möglicherweise Sulfosilikate in den Schlacken enthalten sein könnten, wurde eine Untersuchung unternommen, um diese bis jetzt noch nicht bekannten Verbindungen darzustellen. Hierbei war es nötig, die Darstellungsmethoden des amorphen Siliciums und des Siliciumsulfids einem eingehenden Studium zu unterwerfen. Es stellte sich dabei heraus, dals die in der Litteratur angegebenen Methoden zur Darstellung des amorphen Siliciums der Natur der Versuchsanordnung nach entweder nur in sehr kleinem Malsstabe ausgeführt werden können oder schlechte Ausbeute ergeben.

Berzelius giebt an, dafs man Silicium darstellen kann, indem man Chlorsilicium oder Fluorsilicium über in einem Glasrohr erhitztes Kalium leitet oder indem man ein Gemisch von Kieselfluorkalium und metallisches Kalium in einem Eisenrohre erhitzt. Alle 3 Methoden eignen sich nicht für die Darstellung gröfserer Mengen von Silicium.

${ }^{1}$ Diese Arbeit ist ein Auszug der Inaugural-Dissertation des Dr. von HaAsy an der Universität Rostock. 
Nach WöHLER stellt man Silicium dar, indem man in einen glühenden eisernen Tiegel ein Gemisch von Kieselfluornatrium, Kochsalz und Natrium einträgt. Nach dem Erkalten wird das Reaktionsprodukt zur Entfernung des Natriumfluorids wiederbolt mit salzsäurehaltigem Wasser ausgekocht und das so gewonnene Silicium zuletzt noch mit wässeriger Flufssäure behandelt. Das Verfahren leidet an dem Übelstand, dafs das Natrium mit dem Kieselfluornatrium nicht innig genug gemischt werden kann und nach Beendigung der Reaktion gewöhnlich ein beträchtlicher 'leil des reduzierten Siliciums verbrennt.

Nach Gattermann glüht man ein Gemisch von Quarzsand mit Magnesiumpulver. CI. WinkLer hat gezeigt, dafs es zweckmäisig ist, entweder dem Reaktionsgemisch etwas Magnesia zuzusetzen oder den Quarzsand in Überschuls zu nehmen, um die Bildung von Siliciummagnesium zu vermeiden.

Aus dem Reaktionsprodukt kann Silicium durch Umschmelzen mit Zink direkt krystallinisch erhalten werden oder aber es wird behufs Entfernung von $\mathrm{Mg}$ und $\mathrm{MgO}$ in konzentrierte Salzsäure eingetragen und der Rückstand mit Flufssäure gereinigt.

Die Reinigung des reduzierten Siliciums ist jedoch wegen des Überschusses an Quarzsand sehr schwierig; die Ausbeuten sind gering.

WARREN leitet Fluorsilicium über erhitztes Magnesium, wodurch Silicium und Fluormagnesium gebildet wird. Das Verfahren giebt schlechte Ausbeuten, da das Fluormagnesium zu schwer schmelbar ist.

Mannigfache Versuche, um die vorgeschlagenen Methoden zu verbessern, führten schliefslich zu dem im nachfolgenden beschriebenen Verfahren, welches gestattet, mit Leichtigkeit gröfsere Mengen von Sicilium darzustellen.

Als Grundlage diente die schon von St. Clatre-Deville' vorgeschlagene Reaktion von Fluorsilicium auf Natrium. Es ist dieselbe besonders geeignet, da sich Fluorsilicium leicht darstellen läfst und das Natrium billig und leicht schmelzbar ist.

Vorversuche lehrten, dals die Reaktion zwischen Fluorsilicium und Natrium bei einer Temperatur von $400-500^{\circ}$ gut vor sich geht. Die Reaktion ist dabei so heftig, dafs der Apparat eine Einrichtung haben mufs, die verhindert, dafs durch das Verschwinden des Fluorsiliciums eingesogene Luft mit dem Natrium in Berührung kommen kann. Zu diesem $Z$ weck führt man die Reaktion am besten 
in einem tiefen gufseisernen Tiegel aus, von der Einrichtang, wie Figur zeigt.

Als Fluorsiliciumentwickler dient ein Glasballon $A$ oder ein gufseiserner Topf mit aufgeschliffenem Deckel von 5-6 Litern Inhalt, welcher in einem Sandbade auf einem gewöhnlichen Gasofen steht. Zur Entwickelung des Fluorsiliciums wird am besten ein Gemisch von $750 \mathrm{~g}$ Flulsspat, $1125 \mathrm{~g}$ getrocknetem weifsen Sand und $1500 \mathrm{~g}$ roher konzentrierter Schwefelsäure verwendet. Diese

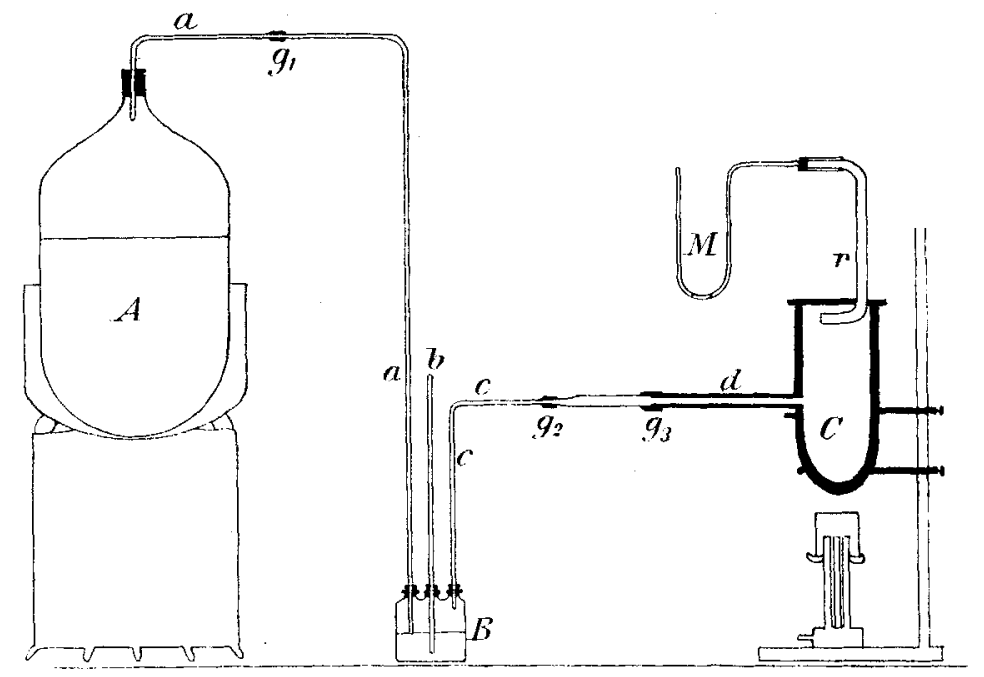

Mischung giebt einen starken Gasstrom. Man ist nur genötigt, am Anfange das Sandbad etwas anzuwärmen, dann bleibt die Entwickelung 3-4 Stunden nahezu konstant. Von $A$ gelangt das Gas durch die Glasröhre $a$ in die WouLfF'sche Flasche $B$. In $B$ ist nur so viel Schwefelsäure, dafs $a$ ganz wenig in dieselbe hineinragt. $b$ ist Druckmesser, um eine während der Operation eingetretene Verstopfung oder Undichtigkeit anzuzeigen. Aus der WouLFF'schen Flasche geht das Fluorsilicium durch die rechtwinkelig gebogene Glasröhre weiter in den Eisentiegel $C$. Der Tiegel ist $21 \mathrm{~cm}$ hoch und hat $8 \mathrm{~cm}$ oberen inneren Durchmesser. $12 \mathrm{~cm}$ iaber dem Boden ist ein Eisenrohr $c$ eingeschraubt. In dem Deckel desselben befindet sich ein doppelt gebogenes Eisenrohr.

Die Dimensionen des Tiegels gestatten gerade, das Reaktionsgemisch von $100 \mathrm{~g}$ Natrium Ausgangsmaterial zu fassen. Das in den Tiegel eingeschraubte fingerdicke Eisenrohr $d$ hat eine Länge 
von $18 \mathrm{~cm}$. Dieselbe ist so ausprobiert, dafs beim Erhitzen des Tiegels $C$ die am Ende der Röhre angebrachte Gummiverbindung $g_{3}$ nicht mehr Gefahr läuft, anzubrennen.

Die Länge des in den Deckel eingeschraubten Eisenrohres $r$ bis zum rechten Winkel ist $21 \mathrm{~cm}$. Der innere Durchmesser dieser Röhre beträgt $1.2 \mathrm{~cm}$.

Im Manometer $M$, das aus einer gewöhnlichen, nicht zu engen, U-förmig gebogenen Glasröhre besteht, befindet sich als Sperrflüssigkeit Petroleum, welches über Natrium gestanden hat; aber nur so viel, dafs gerade der hydraulische Abschlufs erreicht ist.

Bei $g_{1}, g_{2}, g_{3}$ sind Gummiverbindungen, um die Zusammenstellung des Apparates zu erleichtern.

Ist der Apparat so zusammengesetzt, wie ihn die Figur andeutet, so bringt man in den Ballon $A$ die gewogene Menge Schwefelsäure und dann erst das gut durchgearbeitete Gemenge von Flufsspat und Sand, weil anderenfalls das Gemisch von Sand und Flufsspat an den Ballonwänden ankleben würde. Nach dem Eintragen desselben wird der Ballon einige Male kräftig durchgeschüttelt und mit der WouLFF'schen Flasche in Verbindung gebracht. Die Gasentwickelung beginnt sofort und wird durch Anwärmen zu einer lebhaften gesteigert. Zu gleicher Zeit erbitzt man mit einem Bunsenvierbrenner den Eisentiegel $C$ mit aufgesetztem Deckel, aber noch ohne Sperrflüssigkeit im Manometer, um die Eisengefälse ganz trocken zu bekommen. Sobald sich weifse Dämpfe im Manometer zeigen, bringt man die Sperrflüssigkeit in dasselbe und wartet, bis die Dämpfe ganz dicht austreten. Inzwischen ist der Tiegel $C$ so heifs, dafs er am Boden schwach glüht. Ist dieser Punkt erreicht, so hebt man auf kurze Zeit den Deckel, wirft von den vorher abgewogenen $100 \mathrm{~g}$ Natrium ein Stückchen von ungefähr $1 \mathrm{~g}$ rasch hinein und schliefst wieder. Nach ungefähr 1-2 Minuten zeigt das Manometer Unterdruck. Nun mufs man warten, bis sich wieder Überdruck einstellt und beim Manometer dichte weifse Dämpfe austreten. Dann wirft man wieder ein Stückchen Natrium hinein u. s. w. Je voller der Tiegel, um so länger dauert es, bis Reaktion eintritt, so dals man im ganzen zur Umsetzung von $100 \mathrm{~g}$ Natrium vom ersten Einwurf an gerechnet rund 3 Stunden braucht.

Nach dem letzten Eintragen wird im Fluorsiliciumstrom erkalten gelassen, bis der Tiegel $C$ Zimmertemperatur angenommen hat, was 2-3 Stunden dauert. Aus dem kalten Tiegel stöfst man das dunkelbraun aussehende, stark nach Fluorsilicium riechende, 
poröse Reaktionsgemisch mit einem Steinmeilsel heraus, pulvert es rasch ganz grob in einer Porzellanreibschale und bringt es in eine trockene, vorher gewogene Büchse.

Wenn die Darstellung in einem gut ziehenden Abzug ausgeführt wird, so hat man nicht im mindesten unter dem Fluorsiliciumgeruch zu leiden.

Der Gasballon kann 3-4 mal benutzt werden, wenn er sofort nach beendigtem Versuch mit Wasser gereinigt wird.

Andernfalls erstarrt die Masse, so dals sie später nicht mehr entfernt werden kann. Gul'seiserne Töpfe lassen sich zu einer sehr grofsen Anzahl von Operationen verwenden, ohne dafs sie schadhaft werden.

Das so erhaltene Reaktionsgemisch ist aber nicht, wie Deville annimmt, ein Gemenge von Silicium und Fluornatrium, sondern enthält neben diesem sehr grofse Mengen von $\mathrm{Na}_{2} \mathrm{SiFl}_{6}$, da $\mathrm{NaFl}$ ein sehr gutes Absorptionsmittel für $\mathrm{SiFl}_{4}$ ist. Dieser Umstand ist die Ursache, dafs es nicht möglich ist, direkt aus dem Reaktionsgemisch durch Kochen mit Wasser oder Säuren Silicium darzustellen. Das Kieselfluornatrium ist ein so sehr schwer löslicher Körper, so dafs bei dieser Art das Reinigungsverfahren immer enorm grofse Mengen von Silicium oxydiert werden, so dafs die Ausbeuten ganz unbefriedigende sind. Ebenso ist es unmöglich, aus diesem Produkt durch Erhitzen und Überleiten von Chlor Chlorsilicium darzustellen.

Nach sehr vielfachen Versuchen, die zu keinem Resultat führten, zeigte es sich, dals die einfachste Methode, um aus diesem Reaktionsgemisch das Silicium zu gewinnen, darin besteht, dafs man es mit so viel Natrium und Aluminium zusammenschmilzt, dafs das Natrium gerade genügt, um das vorhandene $\mathrm{Na}_{2} \mathrm{SiFl}_{6}$ zu NaFl und $\mathrm{Si}$ umzusetzen und das Aluminium ausreicht, um die Gesamtmenge des Siliciums zu lösen. Während bei dem WöHLER'schen Schmelzprozefs, wo nur $\mathrm{Na}_{2} \mathrm{SiFl}_{6}$ und $\mathrm{Na}$ zusammengeschmolzen wird, erhebliche Mengen des gebildeten Siliciums verbrennen, ist dies bei diesem Schmelzprozesse nicht der Fall, da die Masse grolse Quantitäten von $\mathrm{NaFl}$ enthält und nur relativ wenig Si beim Schmelzen zu reduzieren ist.

Die Reaktion wird zweckmälsig in folgender Weise ausgeführt:

Zunächst berechnet man, wie viel Natrium zur Zersetzung des absorbierten $\mathrm{SiFl}_{4}$ dem Reaktionsgemisch zugesetzt werden muls. Ein Eisentiegel von den im Vorhergehenden angegebenen Dimensionen falst bequem das aus $100 \mathrm{~g}$ Natrium gebildete Reaktions- 
gemisch. Das was das Reaktionsgemisch mehr als $213 \mathrm{~g}$ wiegt, muls als $\mathrm{SiFl}_{4}$ in Rechnung gesetzt werden.

Will man reaktionsfähiges, amorphes Silicium darstellen, so wählt man die Menge des anzuwendenden Aluminiums so, dafs die Silicium-Aluminiumlegierung 15 bis höchstens $16.5 \%$ Si erhält. Macht man die Legierung höherprozentig an Siliciumgehalt, so wird viel krystallisiertes Silicium gebildet, was weniger leicht reagiert. Das Aluminium wird am besten in Form ganz kleiner Schnitzel aus Aluminiumblech angewendet. Diese werden mit der gewogenen Menge Natrium, was ebenfalls in kleine Stückchen geschnitten ist und sich aus dem gefundenen $\mathrm{SiFl}_{4}$-Gehalt der Reaktionsmasse berechnet, gemischt und die so vorbereitete Masse portionsweise in einen hellrotglühenden hessischen Tiegel gebracht, in welchem sich schon eine kleine Menge von geschmolzenem Aluminium befindet.

Den Ofen füllt man so weit mit Holzkohlen und Koks an, dafs der Tiegel bis zum oberen Rand sich in der Glut befindet.

Zum Eintragen hebt man auf kurze Zeit den Deckel, wirft von dem Gemisch einen Blechlöffel voll hinein, schliefst wieder rasch und wartet, bis die Reaktion, die man deutlich hören kann, vorüber ist, bevor man die nächste Portion hineinbringt u. s. w. Wenn das gesamte Gemisch sich im Tiegel befindet, deckt man den Ofen gut zu, legt event. noch etwas Koks nach und wartet, bis die Temperatur so weit gestiegen ist, dafs grofse Natriumflammen aus dem Tiegel herausbrennen. Dann wird derselbe mit Hilfe einer grofsen Zange aus dem Ofen herausgehoben, einige Male vorsichtig auf den Boden aufgeklopft, um die Trennung der metallischen Masse von der Schlacke zu vervollständigen, und erkalten gelassen. Die dünnflüssige Schlacke ist sowohl während des Schmelzprozesses als auch beim Erkalten ein Schutz gegen das Verbrennen von Silicium.

Nach dem Erkalten muls der Tiegel zerschlagen und der Regulus sorgfältigst mechanisch von der anhaftenden Schlacke getrennt werden, was keine Schwierigkeit bietet. Den Regulus löst man in Salzsäure (2 Volumteile Wasser auf 1 Volumteil konzentrierter Salzsäure; bei stärkerer Konzentration wird die Wasserstoffentwickelung zu heftig), das Aluminium geht in Lösung und das Silicium bleibt zurück. Dasselbe wird abgesaugt und noch einmal mit konzentrierter Salzsäure ausgekocht, hierauf wieder abgesaugt, mit Wasser gewaschen, bis keine Salzsäure mehr nachzuweisen ist, und bei $130^{\circ}$ getrocknet. Das auf diese Art dargestellte Silicium besitzt graublaue Farbe. Sein Kieselsäuregehalt schwankt zwischen 3 und $4 \%$. 
Will man das Silicium ganz rein haben, so braucht man es nur mit Flufssäure abzurauchen, wobei allerdings auch wieder etwas Silicium verloren geht.

Die Ausbeute schwankt zwischen $90-95 \%$ an Silicium, welches noch $3-4 \% \mathrm{SiO}_{2}$ enthält.

Durch das Abrauchen mit Flufssäure geht die Farbe von bleigrau in braun iiber und die Ausbeute verringert sich auf $60-62 \%$ der theoretischen.

Es ist so möglich, ein sehr stark reaktionsfähiges Silicium herzustellen, welches schon beim gelinden Erhitzen in der Luft zu Kieselsäure verbrennt.

Durch Zusammenschmelzen relativ gröfserer Mengen von Silicium mit weniger Aluminium gelang es, eine Legierung der beiden Elemente herzustellen, die $22.5 \%$ Silicium enthielt; das daraus gewonnene Silicium war aber weniger reaktionsfähig. Die Gesamtausbeute an Silicium betrug nur 50\%. Hierdurch ist der Beweis erbracht, dals das Silicium genau wie der Kohlenstoff im Eisen ebenfalls in mehreren Modifikationen in seinen Legierungen mit Aluminium auftritt.

Um die beiden so erhaltenen Modifikationen des Siliciums auf ihre Reaktionsfähigkeit zu prüfen, wurde die Temperatur bestimmt, bei welcher dieselben anfangen, im Chlorstrom zu verbrennen.

In einer Verbrennungsröhre wurden zwei Porzellanschiffehen gebracht, von denen das eine mit dem aus $16.5 \%$ igem, das andere mit aus $22.5 \%$ igem Aluminiumsiliciumlegierung erhaltenen Silicium gefüllt war. Zwischen beiden Schiffchen war ein Thermometer so angebracht, dafs es auf der dick mit Magnesia belegten Eisenblechröhre, welche zur Unterstützung des Glasrohres diente, dicht neben der Verbrennungsröhre stand. Dann wurde Chlor über das Silicium geleitet und die Röhre erhitzt. Hierbei zeigte sich, dafs das amorphe Silicium aus der $16.5 \%$ igen Legierung bei $280^{\circ}$ zu brennen anfing, während das krystallinische sich erst bei $340-350^{\circ}$ entzündet. Vergleichende Versuche, wo einmal das erhaltene Silicium im Wasserstoffstrom, das andere Mal einfach in Luft bei $150^{\circ}$ getrocknet wurde, lehrten ferner, dals bei dieser Temperatur durch Luft keine Oxydation zu Kieselsäure erfolgt. Hingegen zeigte sich, dals Flufssäure nicht nur die beigemischte Kieselsäure als Fluorsilicium verflüchtigt, sondern auch das Silicium unter Bildung von Fluorsilicium und Siliciumwasserstoff sehr erheblich angreift.

Zum Zweck der Analyse des erhaltenen Siliciums verfährt man 
am besten in der Weise, dafs man es im Chlorstrom erhitzt und den Teil wiegt, der nicht als $\mathrm{SiCl}_{4}$ verflüchtigt werden kann.

\section{Darstellung des Siliciumsulfids.}

Fremy giebt an, dafs beim Glühen eines Gemisches von Kohle und Kieselsäure in einem Strome von Schwefelwasserstoffgas Siliciumsulfid gebildet wird.

Pierre stellt zunächst Siliciumchlorhydrosulfid, $\mathrm{SiCl}_{3} \mathrm{SH}$, aus Siliciumchlorid und Schwefelwasserstoff dar, welches bei der Destillation in Schwefel und Schwefelsilicium zerfällt.

SABATIER leitet Schwefelwasserstoff über weilsglühendes krystallisiertes Silicium.

Benzelids verbrennt Silicium in Schwefeldampf bei Weifsglut.

Unsere Versuche lehrten, dals alle 3 Methoden in der Form, wie sie in der Litteratur angegeben sind, keine oder sehr schlechte Ausbeuten gaben.

Die Methoden von Fremx, Pierre und Sabatier halten wir für sehr unzweckmäfsig; vielfach abgeänderte Versuche haben schliefslich dazu gefübrt, einen Weg zu finden, auf welchem man durch direkte Vereinigung des Schwefels und Siliciums Schwefelsilicium leicht darstellen kann. Derselbe besteht darin, daif man amorphes, reaktionsfähiges Silicium, möglichst innig mit der 3 fachen Menge Schwefel gemischt, in einem hessischen Tiegel bei $150^{\circ}$ zusammenschmilzt und die so erhaltene Masse in einen auf Rotglut erhitzten hessischen Tiegel, auf welchen mittels Wasserglas und Braunstein ein weites Porzellanrohr angekittet ist, portionenweise einträgt. Eine Minute nach dem Eintragen erfolgt dann unter deutlich hörbarer Reaktion die Vereinigung der Bestandteile. Erfolgt die Reaktion sofort, so ist die Temperatur zu hoch; man mufs sie dann erniedrigen.

Nach vollendetem Eintragen wird die Temperatur etwas erhöht zum Zweck der Trennung des überschüssigen Sehwefels von dem gebildeten Schwefelsilicium. Nach dem Erkalten der Reaktionsmasse ergiebt sich eine zusammengeschmolzene Masse von $\mathrm{SiS}_{2}$, auf welcher in scharf getrennter Schicht der Schwefel sitzt, so dals er leicht mechanisch getrennt werden kann. Das so dargestellte Siliciumsulfid ist 92-95\% ig mit einer Ausbeute von 80-90\% des angewendeten Siliciums. Die Verunreinigungen bestehen aus unverbundenem Silicium und Kieselsäure, die wahrscheinlich zum grölsten Teil aus dem Tiegelmaterial stammt. Aus diesem Rohsiliciumsulfid 
kann leicht chemisch reines Siliciumsulfid dargestellt werden durch Sublimation bei heller Rotglut unter vermindertem Druck in einer Porzellanröhre. Zu diesem Zweck bringt man las Produkt in eine auf der einen Seite geschlossenen Por\%ellanröhre, die man auf der anderen Seite mit einem doppelt durchbohrten Gummistopfen versieht. In die eine Bohrung des Stopfens steckt man ein knieförmig gebogenes langes Glasrohr, welches man in Quecksilber taucht, in die andere eine Glasröhre, welche, durch dazwischen gelegte Chlorcalciumröhren getrennt, mit einer gewöhnlichen Wasserstrahlluftpumpe in Verbindung steht. Bei einem Druck von $60 \mathrm{~mm}$ Queck. silbersäule erfolgt dann bein Erhitzen die Sublimation ohe Schwierigkeiten.

Nach dem Erkalten der Röhre findet sich das $\mathrm{SiS}_{2}$ in schönen langen, weilsen Nadeln, scharf getrennt von einem orangeroten Körper, der aus $\mathrm{SiS}$ besteht, und dem nichtflüchtigen $\mathrm{Si}$ und $\mathrm{SiO}_{2}$.

\section{Darstellung von Siliciumchlorid.}

Vielfache Versuche lehrten, dals ron allen vorgeschlagenen Methoden das direkte Erhitzen amorphen Siliciums im Chlorstrom die besten Ausbeuten liefert. Die Vereinigung erfolgt bei märsiger Rotglut sehr leicht; es wurden Ausbeuten ron etwa 98\% erhalten.

Die Gatrenmanx'sche Methode hat sich trotz ihrer Einfachheit nicht als zweckmälsig erwiesen, da die Ausbeuten trotz sorgfältiger Eimbaltung der vorgeschriebenen Bedingungeu umbefriedigende sind.

\section{Darstellung von Sulfosilikaten.}

WöHLER hat eine Sulfokieselsäure von der Zusammensetzung $\mathrm{H}_{3} \mathrm{Si}_{4} \mathrm{~S}_{5}$ dargestellt. I)ie Ortho- und Metasulfokieselsäuren und deren Salze sind völlig unbekannt.

Eine grofse Zahl fehlgeschlagener Versuche führte schliefslich zur Darstellung des $\mathrm{Na}_{2} \mathrm{SiS}_{3}$ in der Weise, dal's man zuerst durch Zusammenschmelzen von metallischem Natrium mit Schwefel in einem mit kieselsäurefreicr Koble ausgekleideten hessischen Tiegel reines Schwefelnatrium darstellt und dieses dann mit der quantitativen Menge $\mathrm{SiS}_{2}$ in einem Porzellantiegel durch Zusammenschmelzen zur Vereinigung bringt.

Die Koblenauskleidung der Tiegel wird in der Weise hergestellt, dafs man Hol\%kohlenpulver mit Stärkekleister zu einem steifen Brei anrührt und damit die Innenseite der Tiegel mit einer etwa $3 \mathrm{~mm}$ dicken Schicht überzieht. Hierauf werden die Tiegel bei 
$110-120^{\circ}$ getrocknet, mit kleinen Holzkohlenstücken angefüllt und geschlossen zur Rotglut erhitzt. Die so hergerichteten Tiegel zeigen eine Menge Löcher in der Auskleidung. Um diese zu füllen, giefst man in den Tiegel eine konzentrierte Lösung von Zucker und stellt dieselben in Exsiccatoren, die man so lange evacuiert, bis in der Zuckerlösung keine Luftblasen mehr aufsteigen. Dann wird die Lösung ausgegossen, der Tiegel nochmals getrocknet und geglüht.

Die Analyse des so dargestellten metasulfokieselsauren Natrons ergab:

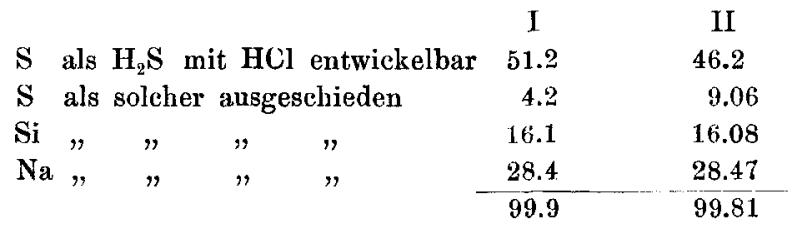

Es ist eine braunschwarze Masse, die beim Übergiefsen mit Wasser in heftigster Weise Schwefelwasserstoff entwickelt; dabei löst sich die Masse vollständig in Wasser. Die so erhaltene Lösung entwickelt auf Zusatz von Säuren keinen Schwefelwasserstoff mehr.

In ganz gleicher Weise wurde versucht, die Sulfosilikate des Magnesiums, Aluminiums und Eisens darzustellen, wobei sich herausstellte, dafs diese Verbindungen, entsprechend deren Karbonaten, durch Glühen in $\mathrm{SiS}_{2}$ und zurückbleibendes Schwefelmetall zerlegt werden können: Führt man die Versuche in kieselsäurehaltigen Gefälsen aus, so erhält man stark mit Kieselsäure verunreinigte Produkte.

\section{Quantitative Untersuchung \\ von schwefelhaltigen Silikaten, Schlacken u. s. w. auf ihren Gehalt an Sulfosilikaten.}

Bringt man Sulfosilikate mit Chlor zusammen, so beobachtet man schon in der Kälte eine sehr starke Einwirkung. Unter starker Wärmeentwickelung entstehen weifse Nebel von Chlorschwefel und Siliciumchlorid. Es ist hierdurch ein Weg gegeben, um Silikate auf ihren Gehalt an Sulfosilikaten zu untersuchen. Zu diesem Zwecke bringt man dieselben in eine Röhre aus schwer schmelzbarem Glas, welche mit geeigneten, mit verdünnter Salzsäure gefüllten Vorlagen versehen sind.

Über die fraglichen Substanzen wird zuerst in der Kälte, später bei Rotglut Chlor geleitet, die in den Vorlagen ausgeschiedene, 
Kieselsäure wird in gewöhnlicher Weise aufgesammelt und nach dem Glühen durch Verflüchtigung mit Flufssäure bestimmt.

Ein Blindversuch ergab, dafs bei den Manipulationen an sich keine Spur von Kieselsäure zu erhalten war.

Auf vorstehend beschriebene Weise wurden der Reihe nach behandelt:

1. Ultramarin (vorher scharf getrocknet).

$3 \mathrm{~g}$ Substanz lieferten $3.4 \mathrm{mg} \mathrm{SiO}_{2}$, dies entspricht $5.21 \mathrm{mg} \mathrm{SiS}_{2}$ oder $0.174 \%, \mathrm{SiS}_{2}$.

2. Hochofenschlacke aus Kladno.

Bei dieser wie auch bei den folgenden Substanzen mulste wegen des Wassergehaltes, welches durch oberflächliche Zersetzung der Silikate in chemisch gebundener Form vorhanden ist, zuerst durch mälsiges Erhitzen in trockenem HCl-Strom getrocknet werden.

$15 \mathrm{~g}$ Substanz lieferten viel Wasser und $4.2 \mathrm{mg} \mathrm{SiO}_{2}$; dies entspricht $6.47 \mathrm{mg} \mathrm{SiS}_{2}$ oder $0.043 \% \mathrm{SiS}_{2}$.

\section{Alterer Lavastrom vom Vesuv.}

$15 \mathrm{~g}$ Substanz lieferten viel Wasser und $0.7 \mathrm{mg} \mathrm{\textrm {SiO } _ { 2 }}$; dies entspricht $1.073 \mathrm{mg} \mathrm{\textrm {SiS } _ { 2 }}$ oder $0.007 \% \mathrm{SiS}_{2}$.

4. Hoch of enschlack e (sehr stark CaS-haltig).

$15 \mathrm{~g}$ Substanz lieferten viel Wasser und $0.9 \mathrm{mg} \mathrm{SiO}_{2}$; dies entspricht $1,38 \mathrm{mg} \mathrm{\textrm {SiS } _ { 2 }}$ oder $0.009 \% \mathrm{SiS}_{2}$.

5. Kupferspurstein.

$5 \mathrm{~g}$ Substanz lieferten viel Wasser und $2.5 \mathrm{mg} \mathrm{SiO}_{2}$; dies entspricht $5.37 \mathrm{mg} \mathrm{SiS}$ oder $0.107 \% \mathrm{SiS}_{2}$.

6. Hochofenschlacke aus Cainsdorf.

$15 \mathrm{~g}$ Substanz lieferten viel Wasser und $10.5 \mathrm{mg} \mathrm{SiO}_{2}$; dies entspricht $15.64 \mathrm{mg} \mathrm{SiS}{ }_{2}$ oder $0.104 \% \mathrm{SiS}_{2}$.

Wenn diese Versuche auch nicht einwandfrei sind, so lassen sie es doch wahrscheinlich erscheinen, dals das Ultramarin, die Lava vom Vesur und viele Schlacken Sulfosilikate enthalten.

Es ist wahrscheinlich, dafs das Auftreten mancher Schwefelquellen in der Natur durch die Zersetzung von sulfosilikathaltigen Gesteinen hervorgerufen wird.

Bei der Redaktion eingegangen am 25. November 1899. 